\title{
Model of tunnelling through periodic array of quantum dots
}

\author{
Dmitry Meynster ${ }^{1}$, Anton Popov ${ }^{l}$, Igor Popov ${ }^{1, *}$ \\ ${ }^{1}$ ITMO University, Department of Higher Mathematics, 197101, St. Petersburg, Russia
}

\begin{abstract}
Several explicitly solvable models of electron tunnelling in a system of single and double twodimensional periodic arrays of quantum dots with two laterally coupled leads in a homogeneous magnetic field are constructed. First, a model of single layer formed by periodic array of zero-range potentials is described. The Landau operator (the Schrodinger operator with a magnetic field) with point-like interactions is the system Hamiltonian. We deal with two types of the layer lattices: square and honeycomb. The periodicity condition gives one an invariance property for the Hamiltonian in respect to magnetic translations group. The consideration of double quantum layer reduces to the replacement of the basic cell for the single layer by a cell including centers of different layers. Two variants of themodel for the double layer are suggested: with direct tunneling between the layers and with the connecting channels (segments in the model) between the layers. The theory of self-adjoint extensions of symmetric operators is a mathematical background of the model. The third stage of the construction is the description of leads connection. It is made by the operator extensions theory method too. Electron tunneling from input lead to the output lead through the double quantum layer is described. Energy ranges with extremely small (practically, zero) transmission were found. Dependencies of the transmission coefficient (particularly, "zero transmission bands" positions) on the magnetic field, the energy of electron and the distance between layers are investigated. The results are compared with the corresponding single-layer transmission.
\end{abstract}

\section{Introduction}

Electron tunnelling through periodic array of quantum dots in a homogeneous magnetic field has been intensively investigated over the last few years because it can be significant for nanotechnology applications [1-6]. Since the publication of famous Hofstadter paper [7] research interest in spectral properties of twodimensional periodic arrays in magnetic field has greatly increased. Fractal structure of the spectrum (and "Hofstadter butterfly" type energy-flux diagrams) has been a theoretical result for long time, but later got experimental confirmation.

There are several different approaches to building models of quantum dot arrays. In this paper zero-range potential model [8-10] based on the theory of self-adjoint extensions of symmetric operators is used [11]. One of its benefits is that the model is explicitly solvable.

Because of high quality of nanostructure devices, large Fermi wave length (i.e. the de Broglie wavelength of electrons with energy close to the Fermi energy) $\left(4 \times 10^{-8} \mathrm{~m}\right)$ and long mean free path of electron $\left(10^{-5}\right.$ $\mathrm{m})$ can be observed [12]. Therefore, one deals with the ballistic regime of electron transport. In this case the Landauer-Buttiker formalism can be used to derive the conductivity $\sigma$ for the nanostructure with several leads from the transmission coefficient $\left.T(E)\right|_{E=E_{F}}$ (here $E_{F}$ is the Fermi energy).
In the simplest case (one incoming and one outgoing channel) the Landauer formula has the form $\sigma=\frac{e^{2} T}{\hbar(1-T)}[13]$. Here $e$ is the electron charge, $\hbar$ is the Plank's constant. The geometry of a nanostructure can influence transmission coefficient a lot.

In this article we consider tunnelling in the system consisting of double two-dimensional periodic array (with square or hexagonal (honeycomb) lattices in each layer) of quantum dots with two connected semi-infinite leads orthogonal to the plane of the array. We study the influence of the magnetic field and tunnelling electron energy on the transmission coefficient and compare our results with the tunnelling through the corresponding single-layer periodic arrays researched earlier [14-15].

\section{Model of single-layer array}

We start from the Hamiltonian of a single particle in constant homogeneous magnetic field $B$. Let us assume that particle has mass $m$ and electric charge $e$. Since the space is $\mathbb{R}^{3}$, we choose a standard basis $\mathbf{i}, \mathbf{j}, \mathbf{k}$ such that $\mathbf{B}$ is collinear to $\mathbf{k}: \mathbf{B}=B \mathbf{k}, B \neq 0$. Then, the particle can move freely along $z$ axis, and the state space of the model is $L^{2}\left(\mathbb{R}^{2}\right)$, where $\mathbb{R}^{2}$ is the plane based on vectors $\mathbf{i}, \mathbf{j}$.

\footnotetext{
* Corresponding author: popov1955@gmail.com
} 
The Hamiltonian $H_{0}$ has the following form:

$$
H_{0}=\frac{1}{2 m}\left(\hat{p}-\frac{e}{c} \mathbf{A}(\mathbf{r})\right),
$$

where $c$ is the speed of light, $\hat{p}=-i \hbar \nabla$ is the twodimensional momentum operator, $\mathbf{A}(\mathbf{r})$ is a vector potential of the field $\mathbf{B}(\mathbf{B}=\operatorname{rot} \mathbf{A})$. Vector potential is not unique, we will use the symmetric gauge $\left(\mathbf{A}(\mathbf{r})=\frac{1}{2} \mathbf{B} \times \mathbf{r}\right)$.

The following standard notations are used:

- $\omega=|e B| / \mathrm{cm}$ is the cyclotron frequency;

- $\Phi_{0}=2 \pi \hbar c /|e|$ is the quantum of the magnetic flux; - $\xi= \pm B / \Phi_{0}$--- number of the flux quanta through the unit area in $\mathbb{R}^{2}$.

The sign of $\xi$ is chosen in such a way that the condition $\xi e B>0$ holds.

We will also use the system of units in which $e=\hbar=m=c=1$, to simplify the calculations. Then, $H_{0}$ can be rewritten in the following form:

$$
H_{0}=-\frac{1}{2}\left[\left(\frac{\partial}{\partial x}+\pi i \xi y\right)^{2}+\left(\frac{\partial}{\partial y}-\pi i \xi x\right)^{2}\right]
$$

We will also need the Green function of $H_{0}$, which has the form $([11])$ :

$$
\begin{aligned}
& G_{0}\left(\mathbf{r}, \mathbf{r}^{\prime}, E\right)= \\
& \frac{1}{2 \pi} \Gamma\left(\frac{1}{2}-\frac{E}{\omega}\right) \exp \left(-\pi i \mathbf{r} \wedge \mathbf{r}^{\prime}-\frac{1}{2} \pi \xi\left\|\mathbf{r}-\mathbf{r}^{\prime}\right\|^{2}\right) \\
& \Phi\left(\frac{1}{2}-\frac{E}{\omega}, 1, \pi \xi\left\|\mathbf{r}-\mathbf{r}^{\prime}\right\|^{2}\right) .
\end{aligned}
$$

Here $\Gamma(x)$ is the Euler Gamma-function, $\Phi(a, c, x)$ is the confluent hypergeometric function of the second kind [6].

To obtain a model for an array of quantum dots, we use the theory of self-adjoint extensions of symmetric operators. We assume that quantum dots are placed in the points of regular two-dimensional lattice $\Xi$. Square and hexagonal lattices are considered in the next sections. We can fix two vectors $\mathbf{a}_{1}, \mathbf{a}_{\mathbf{2}}$ in such a way that any element $\mathbf{l}$ of $\Xi$ has unique representation $\mathbf{I}=\lambda_{1} \mathbf{a}_{1}+\lambda_{2} \mathbf{a}_{2}$, where $\lambda_{1}$ and $\lambda_{2}$ are integers. For the square lattice $a_{1}=(1,0)$ and $a_{2}=(0,1)$ can be used. Note also that the hexagonal lattice can be obtained as a superposition of two offset square lattices:

$$
\Xi=\Xi_{\square}+K
$$

where $\Xi_{\square}$ is square lattice, $K=\{0, \mathbf{b}\}$, $\mathbf{b}=\frac{2}{3}\left(\mathbf{a}_{1}+\mathbf{a}_{2}\right)$.
Now consider the Hamiltonian of the array of quantum dots as a perturbation of operator $H_{0}$. Then, it can be expressed in the following form:

$$
H=H_{0}+\sum_{\mathbf{g}_{\mathbf{i}} \in \Xi} V\left(\mathbf{r}-\mathbf{g}_{\mathbf{i}}\right) .
$$

Due to several physical and mathematical assumptions (namely, we consider separable potential for the lattice with short-range potentials for centers) $[9,12]$, potentials $V$ can be replaced by $\delta$-like one, hence, $H_{0}$ coincides with $H$ everywhere except points of the lattice $\Xi$. To obtain $H$, we first restrict $H_{0}$ onto the set of smooth functions vanishing at the points of the lattice (let the restriction be $S_{0}$ ), and then construct self-adjoint extension of $S_{0}$. The Krein's resolvent formula [8] for infinite deficiency indices describes the difference between the Green functions of $H$ and $H_{0}$ :

$$
\begin{aligned}
& G\left(\mathbf{r}, \mathbf{r}^{\prime} ; \zeta\right)=G_{0}\left(\mathbf{r}, \mathbf{r}^{\prime}, \zeta\right)- \\
& \sum_{\mathbf{g}, \mathbf{g}^{\prime} \in \Xi}[Q(\zeta)+A]_{\mathbf{g}, \mathbf{g}^{\prime}}^{-1} G_{0}(\mathbf{r}, \mathbf{g} ; \zeta) G_{0}\left(\mathbf{g}^{\prime}, \mathbf{r}^{\prime} ; \zeta\right) .
\end{aligned}
$$

Here $Q$ is the Krein Q-function and operator $A$ parameterizes the self-adjoint extension of the operator $H_{0}$.

Finally, we can assume that non-diagonal elements of $Q(\zeta)$ are negligible, hence, it takes the form:

$$
Q(\zeta)_{i j}=q(\zeta) \delta_{i j}
$$

where $\mathrm{q}$ is:

$$
q=-\frac{1}{2 \pi}\left(\psi\left(\frac{1}{2}-\frac{\zeta}{\omega}\right)+\log (\pi \xi)+2 C_{E}\right) .
$$

Here $\psi$ is digamma function and $C_{E}$ is the Euler's constant.

\section{Tunnelling through double layer}

\subsection{Model of tunnelling between layers}

First, we build the model for the square lattice. We assume that the state space of the model is a direct sum of two identical state spaces, one for each layer:

$$
\mathcal{H}=L_{2}\left(\mathbb{R}^{2}\right) \oplus L_{2}\left(\mathbb{R}^{2}\right)
$$

Then, the unperturbed Hamiltonian has the following form:

$$
H_{0}=H_{L} \oplus H_{L},
$$

where $H_{L}$ is the Hamiltonian for the single layer.

To use the Krein's resolvent formula, we, first, restrict operator $H_{L}$ onto the set of smooth functions vanishing at the points of the lattice, let the restricted operator be $S_{L}$. Then, the restriction of $H_{0}$ has the form: 


$$
S_{0}=S_{L} \oplus S_{L} \text {. }
$$

The deficiency space for $S_{0}$ is the direct sum:

$$
\mathcal{G}=\mathcal{G}_{L} \oplus \mathcal{G}_{L}
$$

where $\mathcal{G}_{L}=l_{2}(\Xi)$ is the deficiency space for operator $S_{L}$.

In its turn, the Krein Q-function is the direct sum:

$$
Q=Q_{L} \oplus Q_{L},
$$

where $Q_{L}$ is defined as in (7), (8).

Let $f$ be a function from $l_{2}(\Xi)$, then the Krein $\Gamma$ function of pair $\left(H_{L}, S_{L}\right)$ for a single layer has the following form:

$$
\left(\Gamma_{L}(\zeta) f\right)(\mathbf{r})=\sum_{\mathbf{g} \in \Xi} G_{0}(\mathbf{r}, \mathbf{g} ; \zeta) f(\mathbf{g}),
$$

and the Krein $\Gamma$-function for the whole system can be defined as the direct sum:

$$
\Gamma=\Gamma_{L} \oplus \Gamma_{L} .
$$

Finally, using the Krein resolvent formula for infinite deficiency indices, we obtain an expression for the Green function of $H$ :

$$
\begin{aligned}
& G\left(\mathbf{r}, \mathbf{r}^{\prime} ; \zeta\right)=G_{0}\left(\mathbf{r}, \mathbf{r}^{\prime}, \zeta\right)- \\
& -\sum_{\mathbf{g}, \mathbf{g}^{\prime} \in \Xi \oplus \Xi}[Q(z)+A]_{\mathbf{g}, \mathbf{g}^{\prime}}^{-1} G_{0}(\mathbf{r}, \mathbf{g} ; \zeta) G_{0}\left(\mathbf{g}^{\prime}, \mathbf{r}^{\prime} ; \zeta\right)
\end{aligned}
$$

Now, we need a self-adjoint operator. It is related with the properties of $A$. We assume that the probability of tunnelling between non-adjacent points of the lattice is negligible. Due to the periodicity of the system, the Hamiltonian of a single layer $H_{L}$ should be invariant with respect to the magnetic translation group transformations (see, e.g., [6]), therefore, we have the following property for the terms of operator $A_{L}$ of the single layer:

$$
A_{L_{\mathbf{l}-\mathbf{g}, \mathbf{m}-\mathbf{g}}}=\exp (i \pi \xi(\mathbf{g} \wedge(\mathbf{l}-\mathbf{m}))) A_{L_{\mathbf{l}, \mathbf{m}}} .
$$

As a consequence, it is sufficient to define elements $A_{L_{\lambda, 0}}$ (see [6] for more detailed explanation):

$$
A_{L_{1,0}}=\alpha\left(\delta\left(\lambda_{1}, 0\right)\left(\delta\left(1, \lambda_{2}\right)+\delta\left(-1, \lambda_{2}\right)\right)+\right.
$$$$
\left.+\delta\left(0, \lambda_{2}\right)\left(\delta\left(\lambda_{2}, 1\right)+\delta\left(\lambda_{2},-1\right)\right)\right) \text {. }
$$

Here $\alpha$ is some constant which characterizes the intensity of the interaction.

Now, we need to take into account tunneling between layers, hence, operator $A$ takes the following form:

$$
A=\left[\begin{array}{cc}
A_{L} & \alpha I \\
\alpha I & A_{L}
\end{array}\right]
$$

In the case of hexagonal lattice the main change is different magnetic translations group which lead to changes of the form of operator $A_{L}[15]$ :

$$
\begin{aligned}
& A_{L_{\mathbf{l}_{\mathbf{i}}}, \mathbf{l}_{\mathbf{j}}}=\alpha\left[\delta\left(\mathbf{l}_{\mathbf{i}}-\mathbf{a}_{\mathbf{1}}, \mathbf{l}_{\mathbf{j}}-\mathbf{b}\right)+\right. \\
& \delta\left(\mathbf{l}_{\mathbf{i}}-\mathbf{a}_{\mathbf{2}}, \mathbf{l}_{\mathbf{j}}-\mathbf{b}\right)+ \\
& \delta\left(\mathbf{l}_{\mathbf{i}}-\mathbf{a}_{\mathbf{1}}-\mathbf{a}_{\mathbf{2}}, \mathbf{l}_{\mathbf{j}}-\mathbf{b}\right) \\
& +\exp \left(\frac { 2 } { 3 } i \pi \xi \left(\delta\left(\mathbf{l}_{\mathbf{i}}+\mathbf{a}_{\mathbf{1}}-\mathbf{b}, \mathbf{l}_{\mathbf{j}}\right)-\right.\right. \\
& \left.\left.\delta\left(\mathbf{l}_{\mathbf{i}}+\mathbf{a}_{\mathbf{2}}-\mathbf{b}, \mathbf{l}_{\mathbf{j}}\right)\right)\right) \times \\
& \times\left(\delta\left(\mathbf{l}_{\mathbf{i}}+\mathbf{a}_{\mathbf{1}}-\mathbf{b}, \mathbf{l}_{\mathbf{j}}\right)+\delta\left(\mathbf{l}_{\mathbf{i}}+\mathbf{a}_{\mathbf{2}}-\mathbf{b}, \mathbf{l}_{\mathbf{j}}\right)+\right. \\
& \left.\left.\delta\left(\mathbf{l}_{\mathbf{i}}+\mathbf{a}_{\mathbf{1}}+\mathbf{a}_{\mathbf{2}}-\mathbf{b}, \mathbf{l}_{\mathbf{j}}\right)\right)\right]
\end{aligned}
$$

\subsection{Model of tunnelling}

Now, we would like to build the general model of tunnelling through the quantum layer (single or double, it doesn't matter). To do that, we use the idea from [17]: let $D$ be any device that is connected to a pair of semiinfinite leads at points $C_{-}$and $C_{+}$. We assume that the Green function $G_{D}$ for $D$ is already known. Let $\mathcal{H}_{D}$ be the state space for the device, $\mathcal{H}_{+}$be the state spaces for leads, then, the state space for the whole system is given as follows:

$$
\mathcal{H}_{0}=\mathcal{H}_{-} \oplus \mathcal{H}_{D} \oplus \mathcal{H}_{+} .
$$

The Hamiltonian of the system without interaction between the device and the channels is just the orthogonal sum of the corresponding operators:

$$
H_{0}=H_{-} \oplus H_{D} \oplus H_{+},
$$

where $H_{D}$ is the Hamiltonian of a charged particle in $D, H_{ \pm}$is the Hamiltonian of the charged particle in space $L^{2}\left(R_{+}\right)$under the Neumann condition at the edge (it has the form $-\frac{d^{2}}{d x^{2}}$ ). It is easy to find the Green functions for $H_{ \pm}$:

$$
\begin{aligned}
& G_{ \pm}\left(x, x^{\prime} ; \zeta\right)= \\
& \frac{i}{2 k}\left[\exp \left(i k\left|x-x^{\prime}\right|\right)+\exp \left( \pm i k\left(x+x^{\prime}\right)\right)\right],
\end{aligned}
$$

where $k^{2}=\zeta$.

Using the theory of self-adjoint extensions again, we can obtain the model Hamiltonian $\mathcal{H}$. To do that, we, first, restrict $H_{0}$ onto the set of smooth functions vanishing at the points $C_{ \pm}$, then, build its extension $H$.

After solving the scattering problem for $H$, we obtain the following formula for the transmission coefficient $T(E)$ :

$$
T(E)=\frac{\left|\left(\alpha_{-} \alpha_{+} Q_{21}^{D}\right)^{2}\right|}{E|\operatorname{det}[Q(E)+A]|^{2}} .
$$


Here $A$ is the matrix of the self-adjoint operator that characterizes the extension,

$$
A=\left[\begin{array}{cccc}
0 & \alpha_{-} & 0 & 0 \\
\alpha_{-} & 0 & 0 & 0 \\
0 & 0 & 0 & \alpha_{+} \\
0 & 0 & \alpha_{+} & 0
\end{array}\right],
$$

$Q(E)$ is the Krein Q-function:

$$
\begin{aligned}
& Q(E)= \\
& {\left[\begin{array}{cccc}
Q_{-}(E) & 0 & 0 & 0 \\
0 & Q_{D}^{11}(E) & Q_{D}^{12}(E) & 0 \\
0 & Q_{D}^{21}(E) & Q_{D}^{22}(E) & 0 \\
0 & 0 & 0 & Q_{+}(E)
\end{array}\right],}
\end{aligned}
$$

$Q_{ \pm}(E)=G_{ \pm}(0,0 ; E)$ and $Q_{D}$ is $2 \times 2$ Krein $Q$ function for the extension, where $Q_{D}^{12}(E)=\overline{Q_{D}^{21}(E)}=G_{D}\left(\mathbf{r}_{1}, \mathbf{r}_{2} ; E\right)$

and

$Q_{D}^{11}(E), Q_{D}^{22}(E)$ are the regular parts of $G_{D}\left(r_{1}, r_{1} ; E\right), G_{D}\left(r_{2}, r_{2} ; E\right)$, correspondingly. Values $\alpha_{ \pm}$describe the quality of contacts $C_{ \pm}$. This technique was used in [14] to construct a model of tunneling through single quantum electron layer.

\section{Results and discussion}

For numerical experiments in this section constants $\alpha, \alpha_{-}, \alpha_{+}$were all selected equal to 1 . Only a finite fragment of an infinite lattice is used for calculations since points which are far enough from contacts don't have much influence on transmission coefficient value. For the square lattice, the basic vectors $a_{1}=(1,0), a_{2}=(0,1)$ were used; the hexagonal (honeycomb) lattice is defined using (4).

The transmission coefficient $T$ is calculated as a function of electron energy $E$ for different values of the magnetic field $B$. The resulting function of two values is represented as a contour plot. We assume that the both contacts are in the same lattice cell, calculations show that putting them in different cells of the lattice leads to decreasing of $T$ but doesn't add or remove any other significant effects.

During the investigation of tunnelling through singlelayer lattices, wide zones in the resulting contour plot with very low value of $T$ were discovered ([14], [15]). The widths of these zones are greater than the product of the Boltzmann constant and the temperature value and, thus, this phenomenon is physically measurable. Our calculations show that these zones are preserved both in the square and the hexagonal lattice cases (see Fig. (14)). Plots were built for the model with direct tunnelling between the layers. The model with channels between the layers gives one qualitatively analogous result such zones exist too.

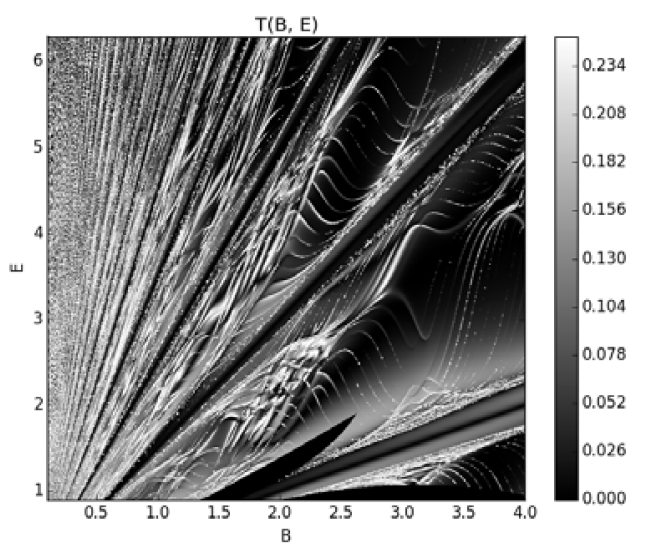

Fig.1. Dependence of transmission coefficient value $T$ on electron energy $E$ and magnetic field $B$ : case of single-layer square lattice. $B$ and $E$ are in dimensionless units.

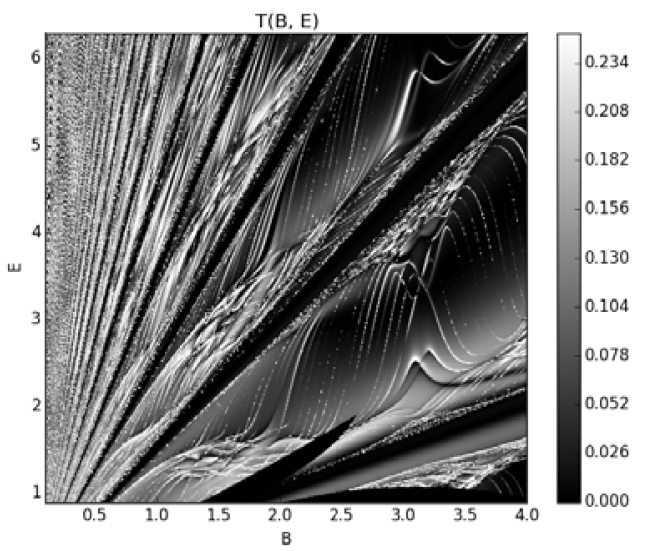

Fig. 2. Dependence of transmission coefficient value $T$ on electron energy $E$ and magnetic field $B$ : case of doublelayer square lattice. $B$ and $E$ are in dimensionless units.

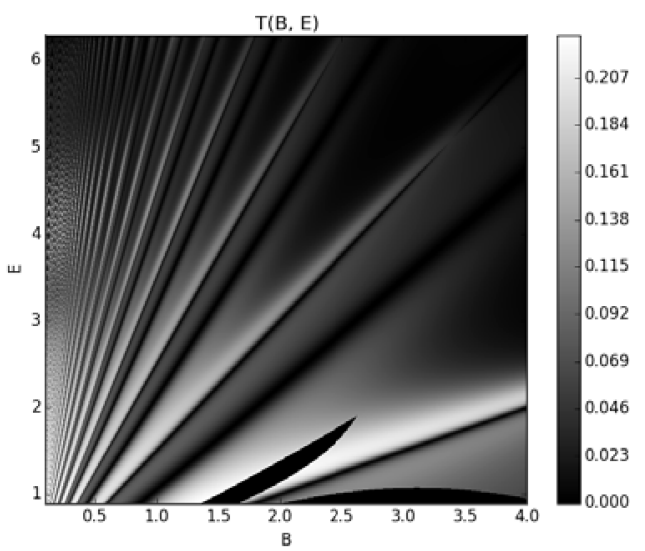

Fig. 3. Dependence of transmission coefficient value $T$ on electron energy $E$ and magnetic field $B$ : case of single-layer hexagonal lattice. $B$ and $E$ are in dimensionless units. 


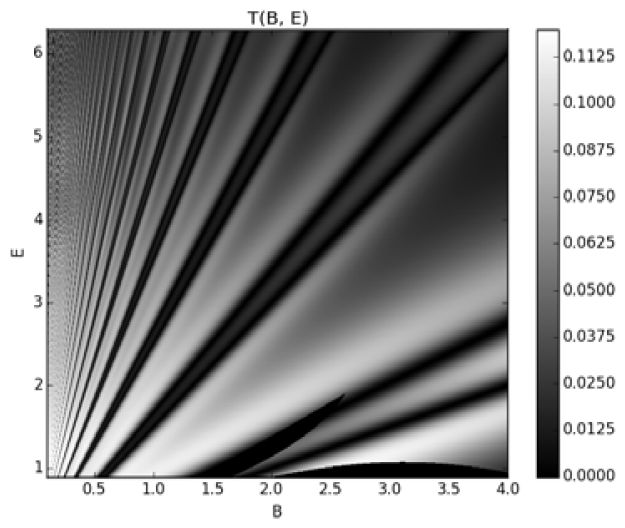

Fig. 4. Dependence of transmission coefficient value $T$ on electron energy $E$ and magnetic field $B$ : case of doublelayer hexagonal lattice. $B$ and $E$ are in dimensionless units.

It can be observed that the middle of each dark strip in Fig. (3) and Fig. (4) coincides with some singularity of $\Gamma\left(\frac{1}{2}-\frac{E}{\omega}\right)$ (which is one of multipliers in (3)).

The dependence of $T$ on $B$ and $E$ shown in the figures is very complicated. For the hexagonal (honeycomb) lattice, it is also complicated but local oscillations of $T$ have less amplitude, that is why in the Fig. (5), (6) it looks like more monotone. Such effect is related to the complex structure of the spectrum of the Hamiltonian for periodic array of quantum dots (see, e.g., [9]). As for the comparison of tunnelling for singlelayer and double-layer cases, one observes that in the model with double layer, dark stripes are wider and inside zones between these stripes, $T$ value is, generally, greater. In the case of the hexagonal lattice, when considering double-layer model, dark stripes pairs are closer to each other and value of $T$ in zones between them is less than in the single-layer case.

This work was partially financially supported by the Government of the Russian Federation (grant 074-U01), by the Ministry of Education and Science of the Russian Federation (GOSZADANIE 2014/190, Projects No 14.Z50.31.0031 and No. 1.754.2014/K)), by grants MK-5001.2015.1 and MK5161.2016.1 of the President of the Russian Federation, by grant 16-11-10330 of Russian Science Foundation.

\section{References}

1. A. Gonzalez, R. Physica E, 10(4), 528-534 (2001)

2. R. Brunner, R. Meisels, F. Kuchar, R. Akis, D.K. Ferry, J.P. Bird, Int. J. Mod. Phys. B., 21, 1288-1296 (2007)

3. M.C. Roco, C.A. Mirkin, and M.C. Hersam. Nanotechnology Research Directions for Societal Needs in 2020. Retrospective and Outlook (Berlin, Springer, 2010)

4. I. Bloch, J. Dalibard, and W. Zwerger, Rev. Mod. Phys., 80, 885-964 (2008)

5. V.A. Geyler, St. Petersburg Mathematical Journal, 3(3), $489-532$ (1992)
6. A. Mantile. Nanosystems: Phys. Chem. Math., 6, 100-112 (2015)

7. D.R. Hofstadter, Phys. Rev.B, 14, 2239-2249 (1976)

8. S. Albeverio and P. Kurasov. Singular perturbations of differential operators. Solvable Schrödinger type operators (London Mathematical Society, London, 2005)

9. V.A. Geyler, B.S. Pavlov and I.Yu. Popov. Atti Sem. Mat. Fis. Univ. Modena, 46, 79-124 (1998)

10. V.A. Geyler, B.S. Pavlov and I.Yu. Popov. J. Math. Phys., 37(10), 5171-5194 (1996)

11. J.P Eisenstein., et.al. Phys. Rev. Lett., 68(9), 1383$1386(1992)$

12. V.A. Geyler, and I.Yu. Popov. Proc. Royal Soc. London A., 454, 697-705 (1998)

13. R. Landauer. IBM J. Res. Dev. 49(24), 17177-17184 (1957)

14. I.Yu. Popov and S.A. Osipov. Chinese Phys. B, 21, 117306 (2012)

15. D.A Eremin, E.N. Grishanov, D.A. Ivanov, A.A. Lazutkina, E.S. Minkin, and I.Yu. Popov. Chinese J. Phys., 52(24), 1100-1109 (2014)

16. V.A. Geyler and I.Yu. Popov, Z. Phys. B, 93, 437439 (1994)

17. V.A. Geyler and I.Yu. Popov. Theor. Math. Phys., 107, 427-434 (1996) 